\title{
An Overview of Comet Composition
}

\author{
Dominique Bockelée-Morvan
}

\author{
LESIA, Observatoire de Paris, F92195, Meudon, France \\ email: dominique.bockelee@obspm.fr
}

\begin{abstract}
Comets are made of ices, organics and minerals that record the chemistry of the outer regions of the primitive solar nebula where they agglomerated $4.6 \mathrm{Gyr}$ ago. Compositional analyses of comets can provide important clues on the chemical and physical processes that occurred in the early phases of Solar System formation, and possibly in the natal molecular cloud that predated the formation of the solar nebula. This paper presents a short review of our present knowledge of the composition of comets. Implications for the origin of cometary materials are discussed.
\end{abstract}

Keywords. comets : general, astrochemistry, Solar System: formation

\section{Introduction}

Comets are remnants of the planetesimals that formed the outer planets. Formed at large distances from the primordial Sun, they have remained for most of their lifetime outside of the orbit of Pluto, either in the trans-Neptunian scattered disk (associated to the Kuiper Belt) or in the Oort cloud. These two dynamically instable comet reservoirs are supplying the ecliptic short-period (also called Jupiter family comets, JFC) and long-period dynamical classes of comets observed in the inner Solar System. Though a variety of subtle evolutionary mechanisms operated for cometary nuclei during their long storage in the Oort cloud and Kuiper belt (Stern 2003) and, for short-period comets, during their many passages close to the Sun, the bulk composition of cometary nuclei is still regarded to be in large part pristine, except possibly for the most volatile ices. Thus, observing comets today provides a window through which we can view an earlier time when the planets were forming. Their composition provides a record of the chemistry of the primitive solar nebula, in the region and at the time of their formation.

Comets have been studied by a number of in situ investigations from spacecraft encounters (Keller et al. 2005), the most recent ones being the Deep Impact and EPOXI missions towards 9P/Tempel 1 and 103P/Hartley 2, respectively (A'Hearn et al. 2005, 2011) and the Stardust mission which collected and returned dust samples of comet $81 \mathrm{P} / \mathrm{Wild} 2$ to Earth for laboratory analyses (Brownlee et al. 2006). Although the in situ measurements have provided important new findings concerning comet composition, the majority of results on the icy component of cometary nuclei have been derived from remote spectroscopic observations of gas phase species in the atmosphere. This paper presents a short review of our present knowledge of the molecular and isotopic composition of comets, focussing on volatiles. Detailed reviews have been published by Bockelée-Morvan et al. (2005), Mumma \& Charnley (2011). Implications on the origin of cometary materials are discussed in Section 6. More detailed discussions are presented in Rodgers \& Charnley (2008), Ehrenfreund et al. (2005) and Irvine et al. (2000). 


\section{Molecular composition of Comets}

\subsection{Molecular inventory of cometary volatiles}

Our present knowledge of the composition of cometary nuclear ices is essentially based on investigations of the coma that develops when comets are approaching the Sun and ices sublimate. Indeed, direct investigations of the nucleus surface (only possible from a spacecraft) only showed the near-infrared spectral signature of water ice on the nucleus of 9P/Tempel 1 (Sunshine et al. 2007), and a still unidentified feature at $2.39 \mu \mathrm{m}$ on that of 19P/Borrelly observed from the Deep Space 1 spacecraft (Soderblom et al. 2002).

About two dozens molecules (not including isotopologues, molecular ions, atoms and radicals) have now been identified in cometary atmospheres (Fig. 1). These identifications were obtained through millimetre, submillimetre and infrared spectroscopy, mass spectrometry aboard the Giotto and Vega spacecraft, and, at a lesser extent, using ultraviolet spectroscopy (see the review of spectroscopic identifications of Bockelée-Morvan et al. 2005). Most identifications were obtained in the exceptionally bright comets C/1995 O1 (Hale-Bopp) and C/1996 B2 (Hyakutake). Observations in the visible wavelength range sample signatures of radicals, atoms and ions that are photolysis and chemical products of parent molecules released from the nuclear ices.

The main components of cometary nuclear ices are water (about $80 \%$ by number) followed by $\mathrm{CO}, \mathrm{CO}_{2}, \mathrm{CH}_{3} \mathrm{OH}, \mathrm{CH}_{4}, \mathrm{H}_{2} \mathrm{~S}$ and $\mathrm{NH}_{3}$ (Fig. 1). A recent investigation of the $\mathrm{CO}_{2}$ abundance in 17 comets using the AKARI space telescope shows that $\mathrm{CO}_{2}$ dominates over CO in most comets (Ootsubo et al. 2010; Hamada et al. 2010). Abundances of detected cometary parent molecules range from less than $0.01 \%$ to $20 \%$ relative to water, and generally decrease with increasing complexity, except for hydrocarbons. Complex species, such as methyl formate and ethylene glycol, are detected. The cumulative histogram of the number of detected species, $N$, as a function of their abundance, $X$, suggests that observational biases affect the detection of species with abundances $<10^{-3}$ and that a large number of molecules remains to be identified in cometary atmospheres (Crovisier et al. 2004).

\subsection{Chemical diversity}

According to current theories, comet formation in the solar nebula extended over a wide range of heliocentric distances for both Oort cloud comets, and Jupiter family comets issued from the trans-neptunian scattered disk. The current vue is that the UranusNeptune zone is the main source region of Oort cloud comets, with some contribution from the Jupiter-Saturn region, while Jupiter family comets formed beyond Neptune. If comets originated from distinct regions of the solar nebula, they could display diversity in their chemical composition depending on the local temperature and nebular composition where they formed. If there was significant mixing of nebular material across large ranges in heliocentric distance, even individual cometary nuclei could exhibit chemical inhomogeneity.

Chemical diversity among comets is indeed observed for both parent volatiles and daughter species. From a study of radicals $\left(\mathrm{OH}, \mathrm{CN}, \mathrm{C}_{2}, \mathrm{C}_{3}, \mathrm{NH}\right)$ in 85 comets, A'Hearn et al. (1995) proposed the existence of two classes of comets, depending on their $\mathrm{C}_{2}$ and $\mathrm{C}_{3}$ abundances: "typical" comets and "carbon-depleted" comets. They found that about one-half of the Jupiter-family comets are $\mathrm{C}_{2}$ and $\mathrm{C}_{3}$-depleted, but the fraction of carbon-depleted Oort cloud comets is much smaller. An update of this study confirming these two compositional classes is presented by Schleicher (2008). Note that the parent of $\mathrm{C}_{2}$ radical is likely $\mathrm{C}_{2} \mathrm{H}_{2}$ (via $\mathrm{C}_{2} \mathrm{H}$ ), but the origin of $\mathrm{C}_{3}$ remains unclear (Helbert et al. 2005). 


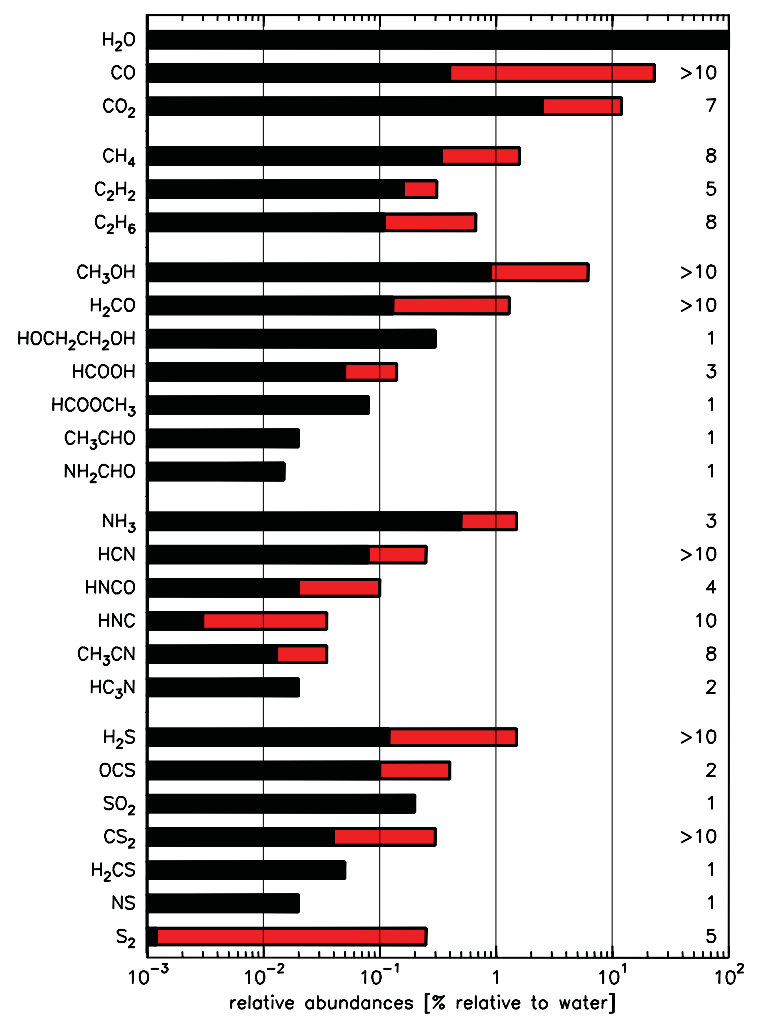

Figure 1. Abundances relative to water of cometary parent molecules. The range of measured values is shown in the gray (red in the color version) portion. The number of comets in which the molecule has been detected is given in the right (adapted from Bockelée-Morvan et al. (2005), courtesy J. Crovisier).

Surveys of parent volatile abundances show strong evidence for chemical diversity among comets. Depending on the molecule, abundances vary by a factor of a few to several tens (for CO) among comets (Fig. 1,2). Diversity is observed both within the population of Oort cloud comets and Jupiter family comets (Fig. 2). No obvious correlation is observed between the abundances of the hypervolatile species and the dynamical class. No trend between volatility and comet-to-comet variability is observed either (Biver et al. 2002; Gibb et al. 2003; Disanti \& Mumma 2008). Three compositional classes, based on the organic composition ( $\mathrm{HCN}, \mathrm{C}_{2} \mathrm{H}_{2}, \mathrm{C}_{2} \mathrm{H}_{6}$ and $\mathrm{CH}_{3} \mathrm{OH}$ ), are possibly emerging (Mumma et al. 2008). Enriched and severely organic-depleted comets are present in both reservoirs (Mumma et al. 2008).

So far, Jupiter family comets have been still poorly investigated, because of their relatively low level of activity. A number of molecules have only been detected in comet C/1995 O1 (Hale-Bopp) or in a small number of comets.

Questions arise whether the molecular abundances measured in the coma are representative of those inside the nucleus, and to which extent the nucleus composition has been altered since comet formation. The chemistry could have evolved both during the long storage within the Oort cloud or Kuiper belt as well as during close passages to the Sun after removal from these reservoirs. Evolutionary effects are most important in the outer layers of a comet while the interior of the nucleus should be in large part 

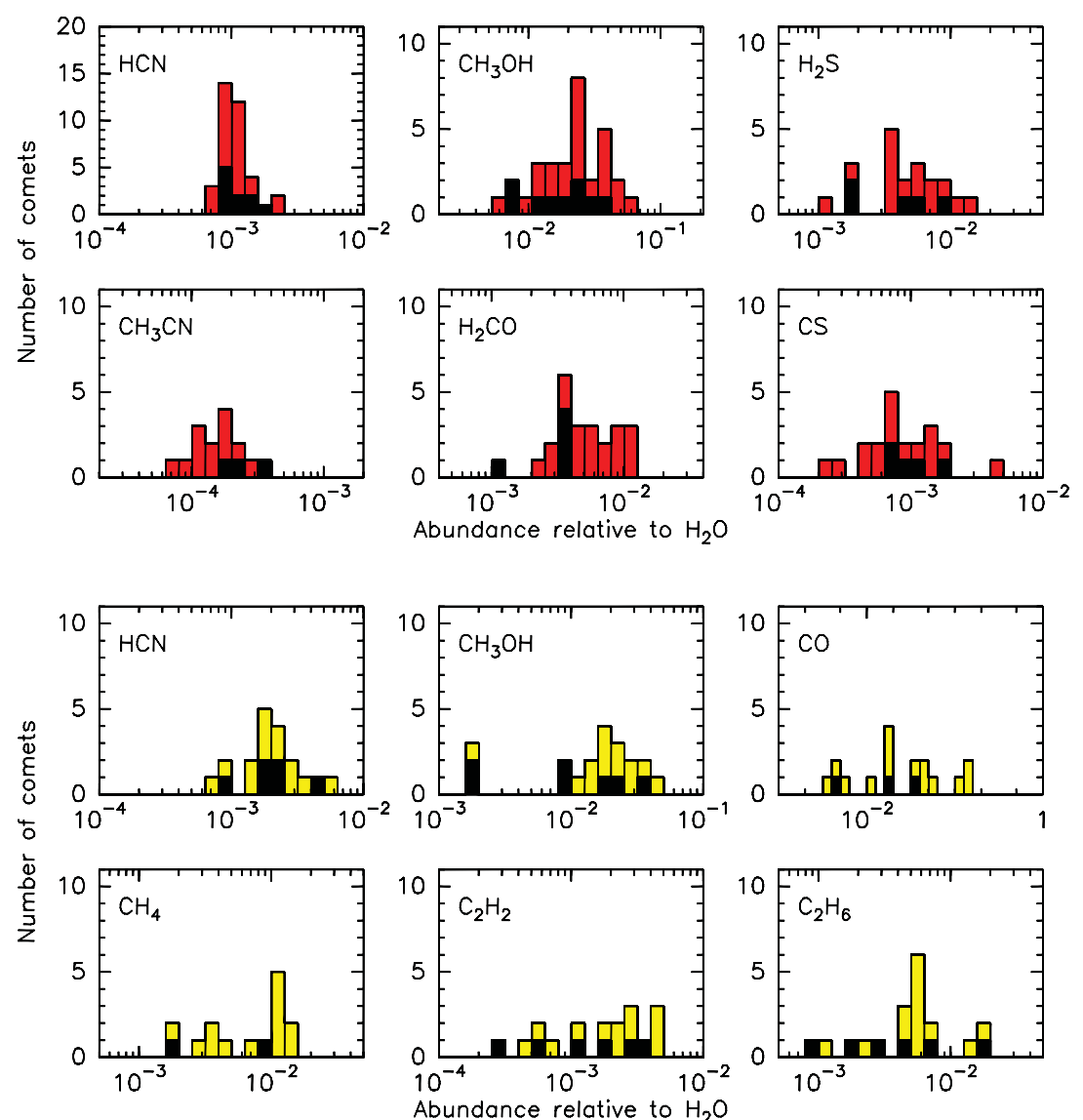

Figure 2. Histogram of molecular abundances relative to water from radio observations (top, Crovisier et al. 2009) and IR observations (bottom). The histogram for Jupiter family comets is in black.

pristine except for perhaps the most volatile species. The consistency of the volatile composition of $73 \mathrm{P} /$ Schwassmann-Wachmann 3 as a function of date and in its individual fragments suggests that evolutionary effects are small (Dello Russo et al. 2007). This might be related to the very low thermal conductivity of surface layers (Groussin et al. 2007; Gortsas et al. 2011). The fact that short-period and long-period comets display similar composition diversity, even in highly volatile species (the exception being CO, which is found abundant only in long-period comets) argues for the composition diversity to be largely pristine.

\section{Isotopic composition of volatiles}

Isotopic ratios are important diagnostics of how cometary materials formed because isotopic fractionation is very sensitive to chemical and physical conditions. So far, only a few isotopic ratios have been measured in cometary gases as summarized in Table 1 (see Jehin et al. 2009, for an extended review).

HDO has been observed from submillimetre spectroscopy in the bright comets HaleBopp and C/1996 B2 (Hyakutake), from which a $\mathrm{D} / \mathrm{H}$ ratio of $3 \times 10^{-4}$ consistent with 
Table 1. Isotopic abundances in cometary volatiles

\begin{tabular}{|c|c|c|c|c|}
\hline Isotopic ratio & Species & Value & Comet & References \\
\hline $\mathrm{D} / \mathrm{H}$ & {$\left[\mathrm{H}_{2} \mathrm{O}\right]$} & $\begin{array}{c}(3.06 \pm 0.34) \times 10^{-4} \\
(2.9 \pm 1.0) \times 10^{-4} \\
(3.3 \pm 0.8) \times 10^{-4} \\
(2.5 \pm 0.7) \times 10^{-4} \\
(4.1 \pm 1.5) \times 10^{-4} \\
(4.6 \pm 1.4) \times 10^{-4} \\
<2.5 \times 10^{-4} \\
(1.61 \pm 0.24) \times 10^{-4} \\
(2.3 \pm 0.4) \times 10^{-3}\end{array}$ & $\begin{array}{l}\text { 1P/Halley } \\
\text { C/1996 B2 (Hyakutake) } \\
\text { C/1995 O1 (Hale-Bopp) } \\
\text { C/2002 T7 (LINEAR) } \\
\text { 8P/Tuttle } \\
\text { C/2001 Q4 (NEAT) } \\
\text { 153P/Ikeya-Zhang } \\
\text { 103P/Hartley 2 } \\
\text { C/1995 O1 (Hale-Bopp) }\end{array}$ & $\begin{array}{l}{[1][2]} \\
{[3]} \\
{[4]} \\
{[5]} \\
{[6]} \\
{[7]} \\
{[8]} \\
{[9]} \\
{[10]}\end{array}$ \\
\hline${ }^{12} \mathrm{C} /{ }^{13} \mathrm{C}$ & $\begin{array}{l}{\left[\mathrm{C}_{2}\right]} \\
{[\mathrm{CN}]} \\
{[\mathrm{HCN}]}\end{array}$ & $\begin{aligned} 93 & \pm 10 \\
91 & \pm 4 \\
111 & \pm 12 \\
114 & \pm 26\end{aligned}$ & $\begin{array}{l}4 \text { comets } \\
21 \text { comets } \\
\mathrm{C} / 1995 \mathrm{O} 1 \text { (Hale-Bopp) } \\
17 \mathrm{P} / \text { Holmes }\end{array}$ & $\begin{array}{l}{[11]} \\
{[12]} \\
{[13]} \\
{[14]}\end{array}$ \\
\hline${ }^{14} \mathrm{~N} /{ }^{15} \mathrm{~N}$ & $\begin{array}{l}{[\mathrm{HCN}]} \\
{[\mathrm{CN}]}\end{array}$ & $\begin{array}{c}205 \pm 70 \\
139 \pm 26 \\
141 \pm 29 \\
220 \pm 40\end{array}$ & $\begin{array}{l}\mathrm{C} / 1995 \mathrm{O} 1 \text { (Hale-Bopp) } \\
17 \mathrm{P} / \text { Holmes } \\
21 \text { comets } \\
73 \mathrm{P} \text { (fragment } \mathrm{C})\end{array}$ & $\begin{array}{l}{[14]} \\
{[14]} \\
{[12]} \\
{[12]}\end{array}$ \\
\hline${ }^{16} \mathrm{O} /{ }^{18} \mathrm{O}$ & {$\left[\mathrm{H}_{2} \mathrm{O}\right]$} & $\begin{array}{l}518 \pm 45 \\
470 \pm 40 \\
530 \pm 60 \\
425 \pm 55\end{array}$ & $\begin{array}{l}\text { 1P/Halley } \\
1 \mathrm{P} / \text { Halley } \\
4 \text { comets } \\
\mathrm{C} / 2002 \mathrm{~T} 7 \text { (LINEAR) }\end{array}$ & $\begin{array}{l}{[2]} \\
{[1]} \\
{[15]} \\
{[5]}\end{array}$ \\
\hline${ }^{32} \mathrm{~S} /{ }^{34} \mathrm{~S}$ & $\begin{array}{l}{[\mathrm{CS}]} \\
{[\mathrm{CS}]} \\
{\left[\mathrm{S}^{+}\right]} \\
{\left[\mathrm{H}_{2} \mathrm{~S}\right]}\end{array}$ & $\begin{array}{l}27 \pm 3 \\
16 \pm 3 \\
23 \pm 6 \\
16 \pm 3\end{array}$ & $\begin{array}{l}\text { C/1995 O1 (Hale-Bopp) } \\
\text { 17P/Holmes } \\
\text { 1P/Halley } \\
\text { C/1995 O1 (Hale-Bopp) }\end{array}$ & $\begin{array}{l}{[13]} \\
{[16]} \\
{[17]} \\
{[18]}\end{array}$ \\
\hline
\end{tabular}

[1] Eberhardt et al. (1995); [2] Balsiger et al. (1995); [3] Bockelée-Morvan et al. (1998); [4] Meier et al. (1998a); [5] Weaver et al. (2011); [6] Villanueva et al. (2009); [7] Hutsemékers et al. (2008); [8] Biver et al. (2006); [9] Hartogh et al. (2011); [10] Meier et al. (1998b); [11] Wyckoff et al. (2000); [12] Manfroid et al. (2009); [13] Jewitt et al. (1997); [14] Bockelée-Morvan et al. (2008); for Hale-Bopp, revised value from the data set of Jewitt et al. (1997) and Ziurys et al. (1999); [15] Biver et al. (2007); [16] Biver et al. (2008); [17] Altwegg (1996); [18] Crovisier et al. (2004); .

Giotto measurements in comet 1P/Halley has been derived (Table 1). More recently, similar values were obtained in other Oort cloud comets from observations of D, OD, and HDO in the UV, near UV, and near infrared, respectively. This is about twice higher than the $\mathrm{D} / \mathrm{H}$ value in terrestrial water, and approximately one order of magnitude larger than the protosolar value in $\mathrm{H}_{2}\left(\sim 2 \times 10^{-5}\right)$ (Fig. 3). The upper limit of $2.5 \times$ $10^{-4}$ measured in comet $153 \mathrm{P} /$ Ikeya-Zhang might suggest that the $\mathrm{D} / \mathrm{H}$ ratio is variable among comets (Biver et al. 2006). DCN has been detected in comet Hale-Bopp, yielding a D/H ratio in HCN seven times higher than that measured in cometary water (Table 1). Measurements of deuteration in other molecules have been attempted (Crovisier et al. 2004). An upper limit of $5 \times 10^{-3}$ was derived for the $\mathrm{D} / \mathrm{H}$ in $\mathrm{CH}_{4}$ (Bonev et al. 2009; Kawakita and Kobayashi 2009). Deuterium enrichments are characteristic of interstellar or protosolar chemistry, and result from ion-molecule or grain-surface reactions at low temperature (Ehrenfreund et al. 2005). 


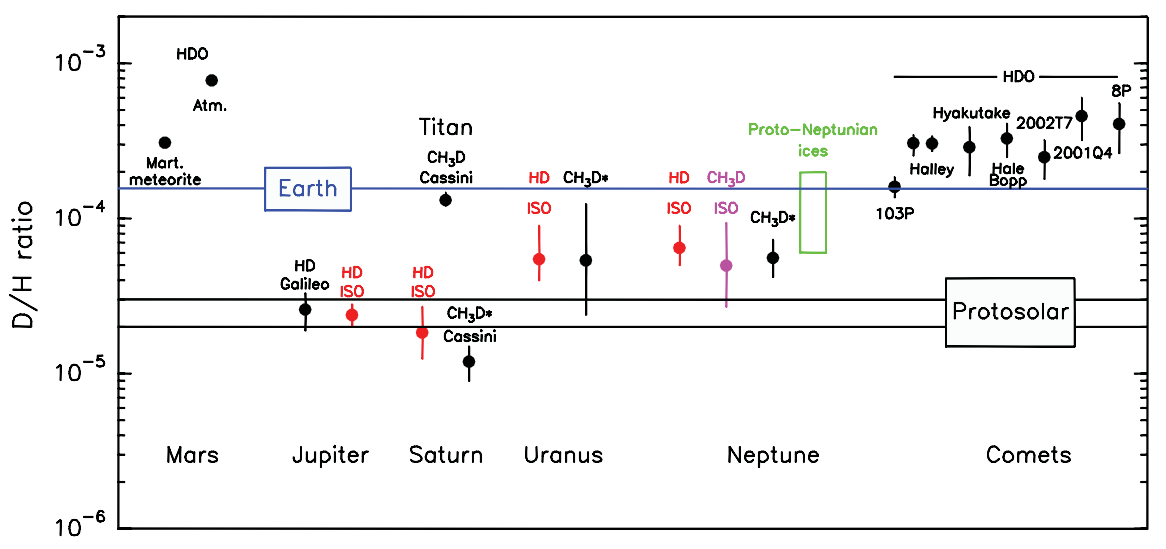

Figure 3. D/H in the Solar System.

The detection of deuterated molecules in the atmospheres of Jupiter family comets is difficult because of their faintness. HDO was detected in the JFC 103P/Hartley 2 with Herschel, from which a $\mathrm{D} / \mathrm{H}$ value equal to the Earth value was derived (Hartogh et al. 2011). Hence, all comets do not share the same $\mathrm{D} / \mathrm{H}$.

Measurements have been obtained for the ${ }^{12} \mathrm{C} /{ }^{13} \mathrm{C}$ ratio in $\mathrm{C}_{2}, \mathrm{CN}$ and $\mathrm{HCN}$, for the ${ }^{32} \mathrm{~S} /{ }^{34} \mathrm{~S}$ ratio in $\mathrm{CS}, \mathrm{S}^{+}, \mathrm{H}_{2} \mathrm{~S}$, and for the ${ }^{16} \mathrm{O} /{ }^{18} \mathrm{O}$ ratio in water: in first approximation they are consistent with terrestrial values $\left({ }^{12} \mathrm{C} /{ }^{13} \mathrm{C}=89 ;{ }^{32} \mathrm{~S} /{ }^{34} \mathrm{~S}=23 ;{ }^{16} \mathrm{O} /{ }^{18} \mathrm{O}=499\right)$. Some measurements suggest an excess in ${ }^{18} \mathrm{O}$ and ${ }^{34} \mathrm{~S}$ (Table 1 ).

The ${ }^{14} \mathrm{~N} /{ }^{15} \mathrm{~N}$ ratio has been measured in the $\mathrm{CN}$ radical in $\sim 20$ comets (Arpigny et al. 2003; Hutsemékers et al. 2005; Manfroid et al. 2005; Jehin et al. 2004; Manfroid et al. 2009). The average value is ${ }^{14} \mathrm{~N} /{ }^{15} \mathrm{~N}=148 \pm 6$, with no significant differences emerging between Oort cloud and Jupiter family comets. Only comet 73P/SchwassmannWachmann 3 presents a value which is out of the typical range $(210 \pm 50$ and $220 \pm 40$, for fragment B and C, respectively, Manfroid et al. 2009). The average value corresponds to a factor of two enrichment in ${ }^{15} \mathrm{~N}$ with respect to the Earth atmospheric value ${ }^{14} \mathrm{~N} /{ }^{15} \mathrm{~N}$ $=272$. The ${ }^{14} \mathrm{~N} /{ }^{15} \mathrm{~N}$ ratio was measured in $\mathrm{HCN}$ in comets $\mathrm{C} / 1995 \mathrm{O} 1$ (Hale-Bopp) and 17P/Holmes, belonging to the Oort cloud and JFC classes, respectively. For comet HaleBopp, an $\mathrm{HC}^{14} \mathrm{~N} / \mathrm{HC}^{15} \mathrm{~N}$ ratio marginally higher than the terrestrial value was reported (Jewitt et al. 1997; Ziurys et al. 1999), but a reanalysis of the data suggests instead a value consistent with the value in $\mathrm{CN}$ (Bockelée-Morvan et al. 2008). The $\mathrm{HC}^{14} \mathrm{~N} / \mathrm{HC}^{15} \mathrm{~N}$ ratio measured in comet $17 \mathrm{P} /$ Holmes is also consistent with the value measured in $\mathrm{CN}$ (Table 1)(Bockelée-Morvan et al. 2008). There is some observational evidence that HCN photolysis is not the sole production mechanism of CN radicals. Indeed, for some comets, the $\mathrm{CN}$ production rate exceeds the $\mathrm{HCN}$ production rate (Fray et al. 2005). CN radicals are also produced by $\mathrm{HC}_{3} \mathrm{~N}$ and $\mathrm{CH}_{3} \mathrm{CN}$ but those species are minor constituents of cometary atmospheres (Fig. 1). This led to the hypothesis that significant amounts of $\mathrm{CN}$ radicals could be produced by the thermal degradation of refractory organics present in dust grains. The similar isotopic properties of $\mathrm{CN}$ and $\mathrm{HCN}$ point to HCN being the prime parent of $\mathrm{CN}$ in cometary atmospheres, or imply that refractory organics producing $\mathrm{CN}$ radicals are equally enriched in ${ }^{15} \mathrm{~N}$.

${ }^{15} \mathrm{~N}$ excesses observed in $\mathrm{CN}$ and $\mathrm{HCN}$ are similar to values measured in carbonaceous meteorites and Interplanetary Dust Particles (IDPs) (Floss et al. 2006; Busemann et al. 


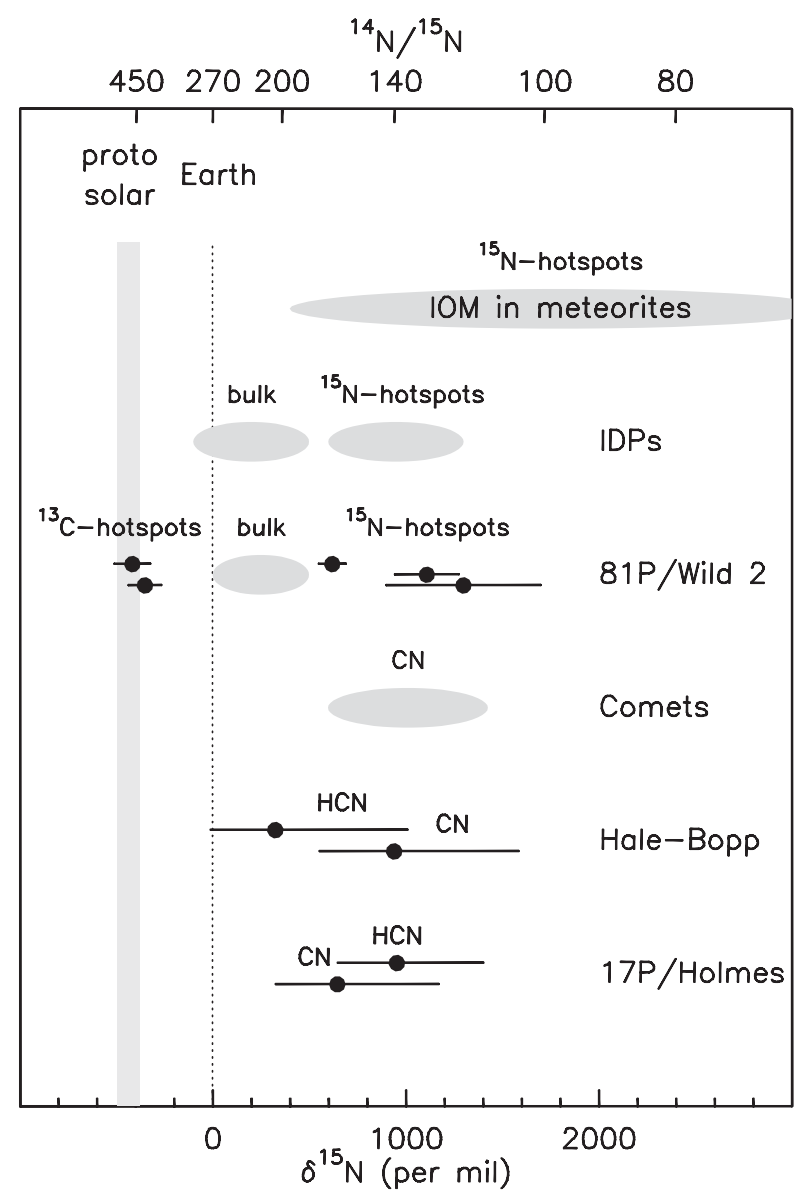

Figure 4. $\mathrm{N}$ isotope measurements in primitive matter of the Solar System, given either as ${ }^{14} \mathrm{~N} /{ }^{15} \mathrm{~N}$ ratios or $\delta^{15} \mathrm{~N}$ values $\left(\delta^{15} \mathrm{~N}=\left({ }^{14} \mathrm{~N} /{ }^{15} \mathrm{~N}\right) /\left({ }^{14} \mathrm{~N} /{ }^{15} \mathrm{~N}\right)\right.$ Earth -1$)$. Shown are the protosolar value measured by the Genesis mission (Marty et al. 2011), values for comet $17 \mathrm{P} /$ Holmes (Bockelée-Morvan et al. 2008), comet Hale-Bopp (Arpigny et al. 2003; Bockelée-Morvan et al. 2008 ) and other comets (Jehin et al. 2009), dust particles of comet 81P/Wild 2 collected by the Stardust mission (McKeegan et al. 2006), interplanetary dust particles IDPs (Floss et al. 2006), and insoluble organic matter (IOM) in carbonaceous meteorites(Busemann et al. 2006). Socalled hotspots are regions that present strong isotopic enrichments relative to the surrounding material. ${ }^{13} \mathrm{C}$ hotspots refer to $81 \mathrm{P} /$ Wild 2 submicrometre grains enriched in ${ }^{13} \mathrm{C}$ with respect to the terrestrial ${ }^{12} \mathrm{C} /{ }^{13} \mathrm{C}$ ratio and depleted in ${ }^{15} \mathrm{~N}$. Points with error bars correspond to single measurements. When many measurements are available, ellipses encompassing the range of measured values are drawn. Adapted from Bockelée-Morvan et al. (2010).

2006)(Fig. 4). ${ }^{15} \mathrm{~N}$ excesses are also present at the submicrometre scale in the dust particles collected by the Stardust mission in comet 81P/Wild 2 (McKeegan et al. 2006). Altogether, it indicates that N-bearing volatiles in the solar nebula underwent important $\mathrm{N}$ isotopic fractionation at some stage of Solar System formation.

\section{Ortho-to-para ratios}

Molecules with $\mathrm{H}$ atoms at symmetrical positions may exist in different nuclear-spin species, e.g. ortho and para species for molecules with two $\mathrm{H}$ atoms. The ortho/para 


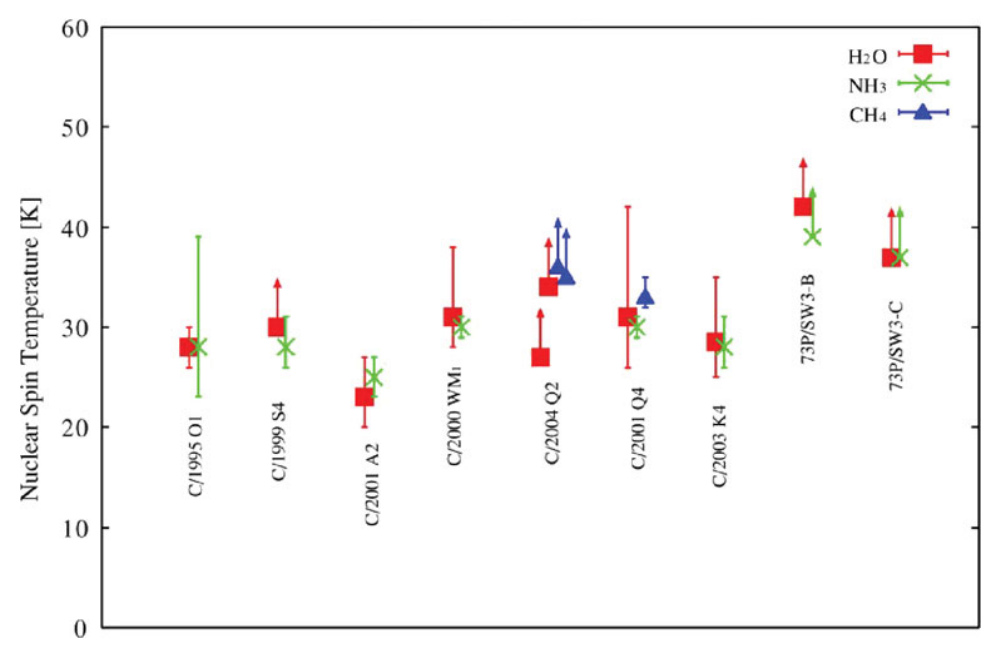

Figure 5. Comparison of nuclear spin temperatures of $\mathrm{H}_{2} \mathrm{O}, \mathrm{NH}_{3}$ and $\mathrm{CH}_{4}$ observed in comets. From Shinnaka et al. (2011).

ratio (OPR) in water and ammonia has been measured in a number of comets (see e.g., Kawakita et al. 2004, Bonev et al. 2007, Shinnaka et al. 2011). Measurements are also available for $\mathrm{CH}_{4}$ (Kawakita et al. 2004) and $\mathrm{CH}_{3} \mathrm{OH}$ (Pardanaud et al. 2007). Most measurements indicate a spin temperature within 25-35 K, though some comets exhibit higher spin temperatures, corresponding to equilibrated OPR. Interestingly, water, ammonia and methane shares the same nuclear spin temperature (Fig. 5). No relationship was found between the spin temperature and the comet dynamical origin, or with the heliocentric distance at which the comets were observed, and their volatile composition. However, there may be some link between the ${ }^{14} \mathrm{~N} /{ }^{15} \mathrm{~N}$ in $\mathrm{CN}$ and the spin temperature of $\mathrm{NH}_{3}$ to be confirmed in the future: fragments $\mathrm{B}$ and $\mathrm{C}$ of comet $73 \mathrm{P} /$ SchwassmannWachmann 3 displays a ${ }^{14} \mathrm{~N} /{ }^{15} \mathrm{~N}$ ratio distinct from the average value (Sect. 3 ) and an equilibrated $\mathrm{OPR}$ in $\mathrm{NH}_{3}$.

It is often argued in the literature that un-equilibrated spin temperatures have a meaning related to the temperature environment of the species in the nucleus or even before, i.e., they could indicate that molecules formed at $\sim 30 \mathrm{~K}$ or were last processed at about 30 K (e.g., Shinnaka et al. 2011). However the real signification of the spin temperature is debated (Crovisier 2007). Buntkowsky et al. (2008) show, by quantum mechanics calculations, that the inter-conversion between para and ortho $\mathrm{H}_{2} \mathrm{O}$ states is very fast inside the ice, and argue that the actual spin temperatures measured in cometary atmospheres depend both on the ice temperature and of the evaporation history of the ice. Thermal desorption is more efficient for para-water than for ortho-water: Dulieu et al. (2011) have estimated to $\sim 2.4\left(T_{\text {spin }} \sim 28 \mathrm{~K}\right)$ the OPR in water set by this process. Further work (both experiments and models) is needed to validate those interpretations with the constraint that the similarity in the spin temperatures of $\mathrm{H}_{2} \mathrm{O}, \mathrm{NH}_{3}$ and $\mathrm{CH}_{4}$ should be explained.

\section{Dust composition}

First insights on the composition of the refractory component of cometary nuclei were obtained from in situ measurements in comet 1P/Halley. Mass spectrometry analysis of 
cometary dust revealed the presence of both rocky $(\mathrm{Mg}, \mathrm{Si}, \mathrm{Fe}, \mathrm{Ca})$ and organic refractory particles, the so-called CHON organic grains (Jessberger et al. 1988). Amorphous carbon grains were also identified. Altogether, the dust grains of $1 \mathrm{P} / \mathrm{Halley}$ were found to have a chondritic CI-like elemental composition for the most refractory elements, whereas enrichments are observed for the light elements C, H, N (Jessberger et al. 1988). The concentrations of $\mathrm{C}, \mathrm{H}, \mathrm{O}$ and $\mathrm{N}$ could not be determined in the dust grains of comet $81 \mathrm{P} /$ Wild 2 collected by Stardust but most other elements were also found to be in chondritic abundance (Flynn et al. 2006). A notable exception is sulfur, which was found to be depleted. Whether this depletion is real or reflects capture heating is debated (Zolensky et al. 2006).

The mid and far-IR spectra of comets show that silicates in comets are dominated by Mg-rich olivines and pyroxenes and are in two forms : amorphous and crystalline (Hanner et al. 2005). Crystalline silicates are grains that condense at high temperatures or can be formed from the annealing of amorphous silicates at somewhat lower temperature. The scarcity of the crystalline silicates in the interstellar medium indicates that these compounds formed in the inner hot regions of the solar nebula. Other high-temperature minerals are present in comet Wild 2 Stardust samples, such as Calcium Aluminium-rich inclusions (CAIs) or iron sulfides (Zolensky et al. 2006). Amorphous silicates are presumably relics of the presolar cloud and analogous to the glassy silicates grains (GEMS) found in IDPs. GEMS-like material is found in Stardust but was possibly created during capture (Ishii et al. 2008).

Our knowledge of the nature of the refractory organic matter is rather limited. Based on the mass spectra recorded by the Giotto and Vega spacecraft, a number of compounds have been hypothesized (see the review of Cottin et al. 1999). The presence of formaldehyde polymers is proposed to explain the extended distribution of formaldehyde in cometary atmospheres (Cottin et al. 2004). Spectroscopic studies of the $3.4 \mu \mathrm{m} \mathrm{C}-\mathrm{H}$ signature of Stardust samples indicate that the aliphatic chains of the organics are longer than in carbonaceous meteorites, and in the diffuse interstellar medium. They are also significantly longer than in IDPs (Keller et al. 2006). The aromatic fraction of the total organic matter is small, but is rich in both oxygen and nitrogen compared with meteoritic insoluble organic matter (Sandford et al. 2006; Clemett et al. 2010). Glycine and PAHs have been identified in Stardust samples (Elsila, Glavin, \& Dworkin 2009; Clemett et al. 2010).

\section{Implications for the origin of cometary material}

The identification of interstellar-like organic and other exotic gases sublimated from cometary nuclei gives the definite impression that comets preserve a record of the interstellar composition characteristic of the presolar cloud, or that cometary and interstellar molecules formed by similar processes (Irvine et al. 2000; Bockelée-Morvan et al. 2000; Ehrenfreund et al. 2005). The gas phase composition in the interstellar medium depends on many variables, but almost every parent species observed in comets has also been detected in the interstellar medium, either in cold dark molecular clouds or in dense cloud cores where star formation is taking place. The molecular composition of comets, interstellar ices, hot cores and hot corinos are all characterized by the presence of large quantities of saturated/hydrogenated species (e.g. $\mathrm{H}_{2} \mathrm{O}, \mathrm{CH}_{4}, \mathrm{CH}_{3} \mathrm{OH}, \mathrm{NH}_{3}, \mathrm{H}_{2} \mathrm{~S}$ ), coexisting with oxidized molecules (as $\mathrm{CO}, \mathrm{CO}_{2}$ and $\mathrm{SO}_{2}$ ). For interstellar ices, it is believed that this composition traces primarily a combination of ion-molecule and grain-surface processes at low temperature. Complex organic species can then form through energetic processing by UV photons and cosmic rays. Complex species are not abundant enough 


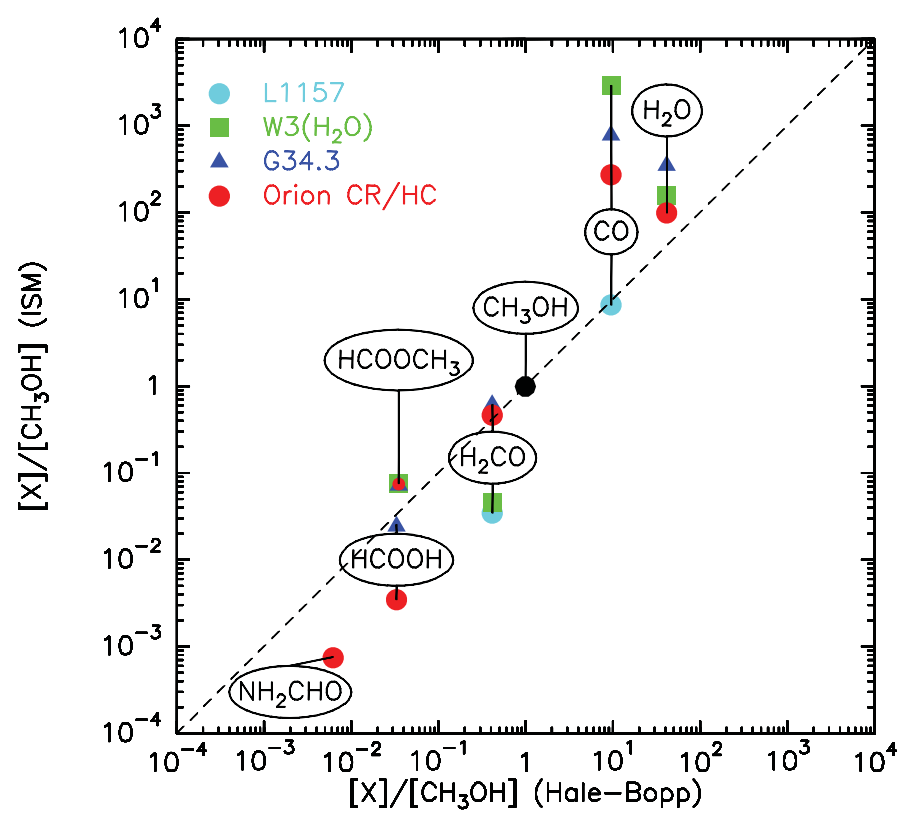

Figure 6. Molecular abundances in comet Hale-Bopp (CHO-bearing species) compared to those in the bipolar flow L1157 and several hot molecular core. From Bockelée-Morvan et al. (2000).

to be detected in interstellar ices, but are found in the hot cores containing massive protostars, or in the 'hot corinos' around low-mass protostars, where the hot environment allows the icy grain mantles to be evaporated. Figure 6 illustrates a quantitative similarity between interstellar and cometary volatile material. Discrepancies do exist. For example, the cometary abundance of ethylene glycol relative to methanol is much larger than measured in the hot core Sgr (B2) (Crovisier et al. 2004). The cometary abundance of methanol is generally much smaller when compared to interstellar ices (Ehrenfreund et al. 2005).

Another indicator of interstellar-like chemistry is the $\mathrm{D} / \mathrm{H}$ ratio, as already discussed in Sect.3. However there is evidence that cometary $\mathrm{D} / \mathrm{H}$ values are lower than in most interstellar sources, in particular around protostars where extreme deuteration is observed (Ceccarelli et al. 2007; Bergin et al. 2010). This low deuterium fractionation in comets is interpreted by ion-molecule chemistry in gas phase at relatively high temperature (25-35 K, Meier et al. 1998a, 1998b) or, alternatively, by mixing of D-enriched material with material reprocessed in the inner solar nebula (Hersant, Gautier, \& Huré 2001). Ion-molecule reactions in the outer solar nebula could also act to lower the interstellar fractionation (Aikawa and Herbst 1999). Extreme D-enrichments are observed in primitive meteorites and IDPs. It was previously thought that these enrichments were reflecting those acquired in the presolar cloud. The negative correlation between the $\mathrm{D} / \mathrm{H}$ ratios of the $\mathrm{CH}$ bonds and their binding energy in the Insoluble Organic Matter of Orgueil carbonaceous meteorite suggests that deuterium enrichments were acquired at the surface of the protosolar disk through intense UV irradiation from the proto-Sun (Remusat et al. 2006). Whether this mechanism affected the D-fractionation observed in cometary $\mathrm{HCN}$ and $\mathrm{H}_{2} \mathrm{O}$ has still to be investigated. It is interesting to note that the 
cometary dust particles collected by Stardust are also less D-enriched than carbonaceous meteorites and IDPs (McKeegan et al. 2006).

The lower $\mathrm{D} / \mathrm{H}$ value found in the water of the Jupiter family comet $103 \mathrm{P} /$ Hartley 2 with respect to Oort cloud comets was not anticipated by models which consider isotopic exchanges in the solar nebula (Hersant, Gautier, \& Huré 2001; Kavelaars et al. 2011). Indeed, these models predict that comets formed at higher distances from the Sun should exhibit higher $\mathrm{D} / \mathrm{H}$ ratios as they incorporated less processed material from the inner solar nebula. Further measurements of the $\mathrm{D} / \mathrm{H}$ ratio in the two dynamical families are required to investigate whether a dichotomy is present, or whether the two families display an isotopic diversity. The current understanding of deuteration in different solar system reservoirs is clearly incomplete.

The isotopic composition of the main carrier of nitrogen in the solar nebula $\left(\mathrm{N}_{2}\right)$ has been recently evaluated to ${ }^{14} \mathrm{~N} /{ }^{15} \mathrm{~N}=441 \pm 6$ from the study of solar wind ions sampled by the Genesis mission (Marty et al. 2011), a value close to measurements in the atmosphere of Jupiter (Fouchet et al. 2004). The interpretation of the ${ }^{15} \mathrm{~N}$ enrichment in cometary $\mathrm{HCN}$ and $\mathrm{CN}$ (a factor of 3 with respect to the protosolar value) is not as compelling as for deuterium. There is indeed still little evidence for $\mathrm{N}$ isotopic fractionation in the interstellar medium (Ikeda et al. 2002; Gerin et al. 2009; Lis et al. 2010), though recent assessments of the $\mathrm{N}$ isotopic fractionation for starless and prestellar cores at various stages of evolution are promising (Milam \& Charnley al. 2011). Rodgers \& Charnley (2008) showed that interstellar chemistry can potentially produce ${ }^{15} \mathrm{~N}$ enrichments in both nitrile and amine groups. On the other hand predicted ${ }^{15} \mathrm{~N}$ enhancements in HCN for exchange reactions in the solar nebula involving the main nitrogen reservoir $\mathrm{N}_{2}$ are modest (Terzieva \& Herbst 2000). Alternative mechanisms include a nucleosynthetic origin and photochemical self-shielding in the solar nebula, as proposed for explaining the oxygen isotope anomalies in meteorites. Irrespective of the mechanism, protosolar HCN never isotopically equilibrated with nebular gas at later phases of Solar System evolution.

The molecular and isotopic compositions of cometary volatiles suggest an origin in chemistry at low temperature. Whether this occurred in the cold molecular cloud that predated the Solar System or in the outer regions of the solar nebula cannot be decided based on the available data. However, the composition of cometary dust shows that cometary nuclei also incorporated nebular products formed in the hot inner regions of the solar nebula, revisiting previous views according to which comets could be pure interstellar condensates (Greenberg 1982). By some process (e.g., turbulence, see Bockelée-Morvan et al. 2002, Ciesla 2007) high-T temperature materials were transported out to the region of formation of comets before their agglomeration, indicating that the solar nebula underwent large-scale radial mixing at early times. Surprisingly, the most recognizable characteristics of presolar material were found to be rare in Stardust samples compared to their occurrence in chondritic porous IDPs, believed to be of cometary origin (Ishii et al. 2008). Only a very few numbers of presolar grains identified by nonsolar isotopic compositions have been found (McKeegan et al. 2006). Altogether, the mineralogical and isotopic composition of comet Wild 2 grains suggests that its refractory material more closely resembles an inner Solar System asteroid than an outer solar system comet with primitive unaltered dust. Likely, comet Wild 2 is not representative of the whole population of comets. Cometary nuclei may present a large diversity in their refractory composition, as observed for their icy composition, relevant to the place and time of their formation in the solar nebula.

Progresses in our knowledge of comet composition will benefit from future space missions to comets (e.g., the Rosetta mission of ESA) and from telescopic observations with 
yet available or new instrumentation (e.g., the Herschel space observatory, ALMA). Our understanding of comet composition in the context of the origin and evolution of our Solar System will greatly benefit from theoretical models of chemical and physical processes in the solar nebula, and from upcoming new results on the composition of protostellar sources and protoplanetary disks at different stages of evolution.

\section{References}

A'Hearn, M. F., Millis, R. L., Schleicher D. G., Osip D. J., \& Birch P. V. 1995, Icarus 118, 223 A'Hearn, M. F., Belton, M. J. S., Delamere, W. A., et al. 2005, Science 310, 258

A'Hearn, M. F., Belton, M. J. S., Delamere, W. A., et al. 2011, Science 332, 1396

Aikawa, Y. \& Herbst, E. 1999, ApJ 526, 314

Altwegg, K. 1996, Habilitationsschrift, University of Bern

Altwegg, K. \& Bockelée-Morvan, D. 2003, SSRv 106, 139

Arpigny, C., Jehin, E., Manfroid, J., et al. 2003, Science 301, 1522

Balsiger, H., Altwegg, K., \& Geiss, J. 1995, JGR 100, 5827

Bergin E. A., Phillips, T. G. \& Comito, C., et al. 2010, A\&\&A 521, L20

Biver, N., Bockelée-Morvan, D., Crovisier, J., et al. 2002, Earth, Moon, and Planets 90, 323

Biver, N., Bockelée-Morvan, D., Crovisier, J., et al. 2006, A\&A 449, 1255

Biver, N., Bockelée-Morvan, D., Crovisier, J., et al. 2007, PשSSS 55, 1058

Biver, N., Bockelée-Morvan, D., Crovisier, J., et al. 2008, LPI Contrib. 1405, 8149

Bockelée-Morvan, D. 2010, EAS 41, 313

Bockelée-Morvan, D., Lis, D. C., Wink, J. E., et al. 2000, A\&A 353, 1101

Bockelée-Morvan, D., Gautier, D., Hersant, F., Huré, J.-M., \& Robert, F. 2002, A $\& A$ 384, 1107

Bockelée-Morvan, D., Crovisier, J., Mumma, M. J., \& Weaver, H. A. 2005, in: M.C. Festou, H.U. Keller, \& H.A. Weaver (eds), Comets II (Univ. of Arizona Press: Tucson), p. 391

Bockelée-Morvan, D., Biver, N., Jehin, E., et al. 2008, ApJ 679, L49

Bonev, B. P., Mumma, M. J., Villanueva, G. L., et al. 2007, ApJ 661, L97

Bonev, B. P., Mumma, M. J., Gibb, E. L., et al. 2009, ApJ 699, 1563

Brownlee, D., Tsou, P., Aléon, J., et al. 2006, Science 314, 1711

Buntkowsky, G., Limbach, H. H., Walaszek, B., et al. 2008, Z. Phys. Chem. 222, 1049

Busemann, H., Young, A. F., O'D. Alexander, C. M., et al. 2006, Science 312, 727

Ceccarelli, C., Caselli P., Herbst E., Tielens A. G. G. M., Caux E. 2007, in: B. Reipurth, D. Jewitt, \& K. Keil (eds), Protostars and Planets V (University of Arizona Press: Tucson), p. 47

Ciesla, F. J. 2007, Science 318, 613

Clemett, S. J., Sandford S. A., Nakamura-Messenger K., Hörz F., McKay D. S. 2010, MESPS 45, 701

Cottin, H., Bénilan, Y., Gazeau, M.-C., \& Raulin, F. 2004, Icarus 167, 397

Cottin, H., Gazeau, M. C., \& Raulin, F. 1999, P\&SS 47, 1141

Crovisier, J. 2007, in: Proceedings of the XVIIIemes Rencontres de Blois: Planetary Science: Challenges and Discoveries, astro-ph/0703785

Crovisier, J., Bockelée-Morvan D., Colom P., et al. 2004, A\&A A 418, 1141

Crovisier J., Biver N., Bockelée-Morvan D., et al. 2009, EMEPP 105, 267

Dello Russo N., Vervack R. J., Weaver H. A., et al. 2007, Nature 448, 172

Disanti M. A., Mumma M. J. 2008, SSRv 138, 127

Dulieu, F. 2011, IAU Symposium 280, this book

Eberhardt, P., Meier, R., Krankowsky, D., \& Hodges, R. R. 1994, A\&A 288, 315

Ehrenfreund, P., Charnley, S. B., \& Wooden, D. 2005, in: M. C. Festou, H. U. Keller, \& H. A. Weaver (eds), Comets II (Univ. of Arizona Press: Tucson), p. 115

Elsila J. E., Glavin D. P., \& Dworkin J. P. 2009, MEPS 44, 1323

Floss, C., Stadermann, F. J., Bradley, J. P., et al. 2006, Geochim. Cosmochim. Acta 70, 2371

Flynn, G. J., Bleuet, P., Borg, J., et al. 2006, Science 314, 1731 
Fouchet, T., Irwin, P. G. J., Parrish, P., et al. 2004, Icarus 172, 50

Fray, N., Bénilan, Y., Cottin, H., Gazeau, M.-C., \& Crovisier, J. 2005, P\&SS 53, 1243

Gerin M., Marcelino N., Biver N., et al. 2009, A\&A 498, L9

Gibb, E. L., Mumma M. J., Dello Russo N., DiSanti M. A., \& Magee-Sauer K. 2003, Icarus 165, 391

Gortsas N., Kührt E., Motschmann U., \& Keller H. U. 2011, Icarus 212, 858

Greenberg, J. M. 1982, in: L.L. Wilkening (ed.), Comets (Univ. of Arizona press: Tucson), p. 1131

Groussin O., A'Hearn, M. F., Li, J.-Y., et al. 2007, Icarus 191, 63

Hamada S., Yamaguchi M., Ootsubo T., et al. 2010, DPS, 42, 962

Hanner, M. S. \& Bradley, J. P. 2005, in: M.C. Festou, H.U. Keller, \& H.A. Weaver (eds), Comets II (Univ. of Arizona Press: Tucson), p. 555

Hartogh, P., Lis, D. C., Bockelée-Morvan, et al. 2011, submitted

Helbert J., Rauer H., Boice D. C., \& Huebner W. F. 2005, A\& A 442, 1107

Hersant F., Gautier D., \& Huré J.-M. 2001, ApJ 554, 391

Hutsemékers, D., Manfroid, J., Jehin, et al. 2005, A\&A 440, L21

Hutsemékers, D., Manfroid J., Jehin E., Zucconi J.-M., \& Arpigny C. 2008, A 6 A 490, L31

Ikeda, M., Hirota, T., \& Yamamoto, S. 2002, ApJ 575, 250

Irvine, W. M., Schloerb, F. P., Crovisier, J., Fegley, B., \& Mumma, M. J. 2000, in: V. Mannings, A.P. Boss, \& S.S. Russel (eds), Protostars and Planets IV (Univ. of Arizona press: Tucson), p. 1159

Ishii, H. A., Bradley, J. P., Dai, Z. R., et al. 2008, Science 319, 447

Jehin, E., Manfroid, J., Cochran, A. L., et al. 2004, ApJ 613, L161

Jehin, E., Manfroid, J., Hutsemékers, D., Arpigny, C., \& Zucconi, J.-M. 2009, EMESP 105, 167

Jessberger, E. K., Christoforidis, A., Kissel, J., et al. 1988, Nature 332, 691

Jewitt, D., Matthews, H. E., Owen, T., \& Meier, R. 1997, Science 278, 90

Kavelaars, J. J., Mousis, O., Petit, J.-M., \& Weaver, H. A. 2011, textitApJ 734, L30

Kawakita, H. \& Kobayashi, H. 2009, ApJ 693, 388

Kawakita, H., Watanabe, J., Furusho, R., et al. 2004, ApJ 601, 1152

Kawakita H., Dello Russo, N., \& Furusho, R., et al. 2006, ApJ 643, 1337

Keller, H. U., Britt, D., \& Buratti, B. J., Thomas N. 2005, in: M.C. Festou, H.U. Keller, \& H.A. Weaver (eds), Comets II (Univ. of Arizona Press: Tucson), p. 211

Keller, L. P., Bajt, S., Baratta, G. A., et al. 2006, Science, 314, 1728

Lis D. C., Wootten A., Gerin M., \& Roueff, E. 2010, ApJ 710, L49

Manfroid, J., Jehin, E., Hutsemékers, D., et al. 2005, A $\mho A$ 432, L5

Manfroid J., Jehin, E., Hutsemékers, D., et al. 2009, A\&SA 503, 613

Marty B., Chaussidon, M., Wiens, R. C., et al. 2011, Science, 332, 1533

McKeegan, K. D., Aléon, J. Bradley, J., et al. 2006, Science 314, 1724

Meier, R., Owen, T. C., Matthews, H. E., et al. 1998a, Science 279, 842

Meier, R., Owen, T. C., Jewitt, D. C., et al. 1998b, Science 279, 1707

Milam, S. \& Charnley, S. B. 2011, IAU Symposium 280, 259P

Mumma, M. J., Disanti M. A., Bonev B. P., et al. 2008, LPI Contrib. 1405, 8282

Mumma, M. J. \& Charnley, S. B. 2011, ARAA in press

Ootsubo, T., Usui, F., \& Kawakita, H., et al. 2010, ApJ 717, L66

Pardanaud, C., Crovisier, J., Bockelée-Morvan, D., \& Biver, N. 2007, in: J.L. Lemaire, \& F. Combes (eds), Molecules in Space and Laboratory, p. 135 (ISBN: 9782901057581).

Remusat, L., Palhol, F., Robert, F., Derenne, S., \& France-Lanord, C. 2006, E\&PSL 243, 15

Rodgers, S. D. \& Charnley, S. B. 2008, MNRAS 385, L48

Sandford, S. A., Aléon, J., Alexander, C. M. O.'D., et al. 2006, Science 314, 1720

Schleicher, D. G. 2008, AJ 136, 2204

Shinnaka, Y., Kawakita H., Kobayashi H., et al. 2011, ApJ 729, 81

Soderblom, L. A., Becker, T. L., Bennett, G., et al. 2002, Science 296, 1087

Stern, S. A. 2003, Nature 424, 639

Sunshine, J., Groussin, O., Schultz, P. H., et al. 2007, Icarus 191, 73 
Terzieva, R. \& Herbst, E. 2000, MNRAS 317, 563

Villanueva, G. L., Mumma, M. J., Bonev, B. P., et al. 2009, ApJ 690, L5

Weaver, H.A., Feldman, P.D., Combi, M.R., et al. Icarus, preprint

Wyckoff, S., Kleine, M., Peterson, B. A., Wehinger, P. A., \& Ziurys, L. M. 2000, ApJ 535, 991

Ziurys, L. M., Savage, C., Brewster, M. A., et al. 1999, ApJ 527, L67

Zolensky, M. E., Zega, T. J., Yano, H., et al. 2006, Science 314, 1735 